\title{
LOS PLANES DE ESTUDIO DE ESPAÑOL EN LAS UNIVERSIDADES DE TAIWÁN
}

\section{The curriculum of Spanish in the universities of Taiwan}

\author{
Margarita NiETo BedoyA \\ Universidad de Valladolid \\ Correo-e: mnieto@flfc.uva.es \\ Mariano Rubia Avi \\ Universidad de Valladolid \\ Correo-e: mrubia@pdg.uva.es \\ Yun Ting Huang \\ Universidad de Valladolid \\ Correo-e: blanche751105@hotmail.com \\ Recepción: I2 de abril de 2016 \\ Envío a informantes: 9 de mayo de 2016 \\ Aceptación definitiva: 25 de julio de 2016
}

Resumen: El gobierno de Taiwán es consciente de que el español es la segunda lengua más hablada en el mundo. La universidad ante tal realidad está asumiendo su enseñanza ya como asignatura optativa o como licenciatura. Las universidades que cuentan con mayor matrícula y con departamento de español son: Fujen, Providence, Tamkang y Wenzao. Ante tal situación hemos considerado importante realizar un análisis sobre sus planes de estudio y hemos llegado entre otras conclusiones a que tienen una orientación estructuralista apoyada en el método tradicional y algo alejada de las recomendaciones del Marco Común Europeo de Referencia para las Lenguas y del Plan Curricular del Instituto Cervantes.

Hoy en los planes de estudio de lenguas extrajeras es fundamental la competencia sociocultural e intercultural, pues sirve al alumnado de puente entre la cultura taiwanesa y la española. Pero los Departamentos de Español de Taiwán se centran más en los contenidos de "Cultura con mayúscula» y ésta no guarda mucha relación con la vida cotidiana, por lo tanto, los estudiantes no pueden comprenderla fácilmente. Así pues, hay que potenciar los aspectos relacionados con la «Cultura con minúscula» y con la «Kultura con k», que giran en torno a los aspectos socioculturales de la cultura meta, temas que potenciarían la competencia intercultural y la competencia comunicativa.

Palabras clave: Taiwán; español; planes de estudio; currículum. 
АвsтRAст: The government of Taiwan is aware that the Spanish is the second most spoken language in the world. University conscious of this fact is assuming its teaching either as an optional subject or degree. The universities that have higher enrollment of students and with the Department of Spanish are: Fujen, Providence, Tamkang and Wenzao. In this situation we have considered important to conduct an analysis of their curriculum and come to other conclusions about the Department of Spanish have a structuralist orientation supported by the traditional method and somewhat away from the recommendations of the Common European Framework of Reference for Languages and the Plan Curricular del Instituto Cervantes.

Today in the curriculum of foreign languages is essential sociocultural and intercultural competence, so that they serve the students as a bridge between Taiwanese culture and Spanish culture. But the Departments of Spanish of Taiwan, focus more on the content of «Culture with a capital C» and this has little to do with everyday life, therefore students can't easily understand. As a result, we must strengthen the aspects related to the «culture with a little c» and «culture with a k» which revolve around the social and cultural aspects of the target culture, themes that would enhance intercultural competence and communicative competence.

KEY wORDs: Taiwan; Spanish; curriculum; curricula.

\section{Introducción: Taiwán y el español}

$\mathrm{T}$

AIWÁN ES UNA ISLA CON UNA GRAN PROYECCIÓN COMERCIAL, dinámica y moderna, en donde la lengua oficial es el chino mandarín junto con los dialectos taiwanés y hakka, y otros idiomas propios de sus diez tribus aborígenes. El interés por el aprendizaje de lenguas extranjeras ha llevado a los jóvenes taiwaneses a estudiar inglés, japonés, español, francés y alemán. El inglés, por su importancia en el mundo, es la lengua más estudiada, siendo obligatoria a partir del $3 .{ }^{\text {er }}$ curso de la enseñanza primaria, después está el japonés, por su influencia geográfica y su avance tecnológico y, en tercer lugar, el español.

La importancia del español se justifica por ser una de las lenguas más habladas en el mundo después del mandarín y el inglés. Moreno Fernández y Otero Roth (2006) afirman que el español es una lengua sólidamente unificada, gracias al elevado número de sus hablantes. En el Informe del Instituto Cervantes $2014^{\mathrm{I}}$, se señala que en el año 2030 el 7,5\% de la población mundial será hispanohablante ( 535 millones de personas), porcentaje que destaca por encima del ruso (2,I\%), del francés (I,I\%) y del alemán (I,I\%). También, se afirma que en el año 2050 Estados Unidos será el primer país hispanohablante del mundo. El Instituto Cervantes (2006) estima que hay 400 millones de hablantes nativos, y roo millones más que hablan el español como segunda lengua, con un total aproximado de 500 millones de hablantes, siendo también la lengua oficial en más de veinte países (España, Hispanoamérica y Guinea Ecuatorial).

El Gobierno de Taiwán es consciente de que el español es la segunda lengua más hablada en todo el mundo, y por lo tanto una herramienta importante para el desarrollo de su economía ya que mantiene relaciones diplomáticas con 12 países hispanoamericanos. España, a través de la Cámara de Comercio y de las oficinas comerciales de

InSTITUto Cervantes (20I4) El español: Una lengua viva. Informe 20I4. Recuperado el I6 de agosto de 20I4, de http://eldiae. es/wp-content/uploads/20I4/o7/El-espa\% $\mathrm{C}_{3} \%$ Biol-lengua-viva-20I4.pdf. 
las Comunidades Autónomas, como la de Cataluña, Valencia y Murcia, tiene relaciones comerciales con Taiwán. Por otra parte, aunque no existe un intercambio directo entre España y Taiwán, varias universidades españolas han establecido relaciones de cooperación e, incluso en algunos casos, imparten programas específicos a grupos de estudiantes taiwaneses que estudian en las universidades españolas para perfeccionar sus conocimientos. Las universidades taiwanesas también mantienen convenios de colaboración con universidades de la América hispanohablante.

Lin (20I2) señala que el Gobierno de Taiwán ha establecido relaciones políticas con países de habla hispana a través de programas como Guidelines for Strengthening Trade with Central and South America Trading Partners, Policy for Strengthening Trade Cooperation with Central America y Action Plan for Strengthening Trade with Europe, para conseguir una buena relación bilateral. Por otra parte, el personal directivo y técnico de las empresas no habla español y, para mejorar sus negocios con Europa e Hispanoamérica, algunas firmas y empresas ofrecen a sus empleados cursos de español en institutos de idiomas.

En cuanto a la situación actual de los graduados en lengua española y su futuro laboral, podemos decir que hablar español suponer tener ciertas ventajas a la hora de solicitar un puesto de trabajo. Los Departamentos de español ofrecen información sobre ofertas laborales en diversos ámbitos (Ministerios de Relaciones Exteriores, Educación, Economía e Información; Oficina de Turismo, Ministerio de Transportes y Comunicación), esto se debe a que del total de los países con los que Taiwán mantiene relaciones diplomáticas, aproximadamente, el 50\% son hispanoamericanos.

El español empezó a hablarse en Taiwán en el año i626 cuando los primeros españoles se instalaron en el norte del país, en ciudades como Keelung, Tamsui e Ilan, donde construyeron escuelas para evangelizar la zona. En aquel periodo, algunos taiwaneses, aunque pocos, aprendieron a leer español. Con el paso del tiempo, religiosos hispanos participaron en la creación de Departamentos de Español en las universidades de Tamkang y en la Católica de Fujen. En el sur, concretamente en Kaohsiung, el agustino recoleto Manuel Piérola fundó una sección de español en el Colegio Universitario de Wenzao, ahora Universidad de idiomas extranjeros Wenzao. Pero a pesar de los años de colonización no se generalizó la lengua española y su enseñanza no se inició de forma sistemática hasta finales del siglo xx.

Actualmente, la mayoría de los universitarios que cursan español lo hacen en cuatro universidades: Fujen, Providence, Tamkang y Wenzao. Las cuatro ofrecen títulos académicos de Licenciatura en Lengua Española y un posgrado con el mayor número de estudiantes y el plan de estudios más significativo y completo. De acuerdo con los datos oficiales del Departamento de Estadística del Ministerio de Educación 20I4, el total de estudiantes universitarios matriculados en los departamentos de español, durante el curso académico 20I3-20I4, incluyendo las secciones nocturna y diurna, era de $2307^{2}$. La mayoría del alumnado termina dedicándose al comercio internacional en empresas privadas, o como traductores e intérpretes requeridos en el sector de servicios (turístico, administración en grandes hoteles, restaurantes y agencias de viajes) y en menor medida en comercios, medios de comunicación, aerolíneas, centros de cultura, etc. Otro grupo importante se dedica a la enseñanza del español

Datos extraídos del 2014 Department of Statistics. Recuperado el I4 de enero de 20I4, de http:// www.edu.tw/pages/detail. aspx ?Node=3752\&Page $=22203 \&$ Index $=7 \& \mathrm{WID}=3 \mathrm{Id} 75 \mathrm{a} 44$-efff-4c44-a075-I5a9eb7aecdf. 
en las universidades o escuelas de idiomas privadas. En los últimos años, debido al incremento de la tendencia del aprendizaje del español como segunda lengua, se ha observado un aumento en la demanda del español en la enseñanza secundaria.

\section{Los Departamentos de Español de las universidades de Fujen, Providence, Tamkang y Wenzao}

En Taiwán existen treinta y dos universidades que imparten español como una de las lenguas propuestas por sus departamentos en formación lingüística. En estos casos se enseña el español bajo la responsabilidad de los departamentos de lenguas extranjeras que tienen profesores de español. En otras facultades como las de Economía, Política, Traducción, etc., el español se ofrece como asignatura optativa o segunda lengua extranjera.

En este trabajo nos vamos a centrar en los departamentos de español de las universidades de Fujen, Providence, Tamkang y Wenzao, pues tienen el mayor número de alumnos. Estos departamentos ofrecen títulos oficiales de Licenciatura en Lengua Española, pero con programas distintos. Fujen, Providence y Tamkang poseen un plan de estudios de 4 años, mientras que Wenzao cuenta con tres programas: College ( 5 años), el Segundo ciclo de la Universidad (2 años) y el Grado de cuatro años (4 años) para los cursos de la sección nocturna y diurna, en el cuadro siguiente se recogen estos datos.

\section{Cuadro i: Departamentos de Español de Fujen, Providence, Tamkang y Wenzao}

\begin{tabular}{|c|c|c|c|c|c|c|c|}
\hline & College & \multicolumn{4}{|c|}{ Licenciatura } & \multirow{2}{*}{$\begin{array}{c}\text { Máster } \\
\mathrm{N}^{\circ} \mathrm{de} \\
\text { estudiantes de } \\
\text { Máster }\end{array}$} & \multirow[b]{2}{*}{$\begin{array}{l}\text { Total de } \\
\text { estudiantes }\end{array}$} \\
\hline Universidad & $\begin{array}{c}\text { Cursos, } \\
\text { grupos y } n^{\circ} \\
\text { de estudiantes }\end{array}$ & $\begin{array}{c}\text { Total de } \\
\text { cursos }\end{array}$ & $\begin{array}{l}\text { Grupos por } \\
\text { curso }\end{array}$ & $\begin{array}{c}\mathrm{N}^{\circ} \text { de } \\
\text { estudiantes } \\
\text { por un grupo }\end{array}$ & $\begin{array}{c}\mathrm{N}^{\circ} \mathrm{de} \\
\text { estudiantes } \\
\text { por } \\
\text { universidad }\end{array}$ & & \\
\hline Fujen & No hay & 4 & 1 & 60 & 285 & 32 & 317 \\
\hline Providence & No hay & 4 & 2 & 50 & 439 & 26 & 465 \\
\hline Tamkang & No hay & 4 & 2 & 60 & 520 & 23 & 532 \\
\hline \multirow[t]{4}{*}{ Wenzao } & & 4 & 2 & 50 & 441 & No hay & 441 \\
\hline & & 4 nocturno & 1 & 45 & 166 & No hay & 166 \\
\hline & & $2\left(2^{\circ}\right.$ ciclo $)$ & 1 & 20 & 31 & No hay & 31 \\
\hline & $\begin{array}{c}5 \text { cursos } \\
\text { (1 grupo } \\
50 \text { alumnos) }\end{array}$ & 5 & 2 & 50 & 264 & No hay & 264 \\
\hline \multicolumn{7}{|c|}{ Total de estudiantes } & 2.216 \\
\hline
\end{tabular}

Cada una de las universidades denomina de manera distinta a sus departamentos de español. Así, para Fujen es Departamento de Lenguas y Culturas Hispánicas; para Providence, Departamento de Lengua y Literatura Española; para Tamkang, Departamento de Español, al igual que para la Universidad de Idiomas Extranjeros de Wenzao.

La importancia de estos departamentos se refleja en el número de alumnos que acogen cada año. El Departamento de Español de Fujen ${ }^{3}$ matricula entre 6o-80 estudiantes cada curso; Providence, cada año académico admite entre Ioo-IIo y los divide en dos grupos; Tamkang, inscribe a I3O-I5o estudiantes, divididos también en dos grupos. En Wenzao, debido a la variedad de modelos educativos, el número de estudiantes

Datos estadísticos ofrecidos por cada universidad. 
varía según cada opción, así el modelo de facultad (cuatro años) posee cada año alrededor de roo estudiantes, 50 por grupo. En líneas generales, cada departamento tiene entre 60-70 estudiantes. De hecho, este número no es el más recomendable para la enseñanza del español pues el método de enseñanza colectivo, que se suele utilizar para el aprendizaje de idiomas, solo se puede llevar a cabo con un número más reducido. Por esta razón, los Departamentos de Español dividen a los alumnos en varios grupos (2 03 ).

El elevado número de matrículas en los departamentos de español nos lleva a cuestionarnos sobre la idoneidad en la ratio profesor/alumno, tan importante para el aprendizaje de un idioma. El español y el taiwanés son culturas muy diferentes que exigen una interacción próxima e intensa entre el profesorado y el alumnado. El Departamento de Español de Fujen tiene una ratio de I:32.47; Providence de i:27.66 y Tamkang de i:24.34. Se excluye el Departamento de Español de Wenzao porque su sistema educativo es diferente al de las otras tres universidades. Si tomamos como ejemplo la clase de conversación y expresión escrita con 20-25 estudiantes por grupo, podemos decir que dicha ratio permite la participación de todo el grupo en las prácticas comunicativas, tanto entre los compañeros como entre el grupo clase y el profesor, potenciando así una metodología activa y participativa. Pero no ocurre lo mismo en las clases que no son prácticas, pues tienen aproximadamente entre 60-70 estudiantes, como por ejemplo las clases de gramática, lectura, cultura, comprensión audiovisual, literatura, cine, turismo, comercio, traducción, historia, geografía, etc. Este número de alumnos por clase hace que el docente trabaje bajo el paraguas de una metodología tradicional en donde el estudiante se limita a esperar, a recibir los conocimientos preparados por el profesorado sin permitir una reflexión, análisis o comparación entre las dos culturas (española-taiwanesa).

La mayor parte de las universidades realizan actividades complementarias. En el caso de los departamentos de español es más relevante ya que tienen como objetivo fortalecer el conocimiento de la cultura española. En el Departamento de Español de Fujen cada año se realizan: obras de teatro, la Semana española y actividades llevadas a cabo por la Asociación de Alumnos del departamento. Providence se centra en: concursos de recitado y teatro, exposiciones de antigüedades, Semana española y un simposio con conferencias impartidas por expertos internacionales. Entre sus actividades extracurriculares se encuentran: el mes del español, donde se realizan exposiciones de cultura española e hispanoamericana, se baila flamenco, hay una Feria del Libro, concurso de teatro, villancicos y competiciones deportivas. Por lo que respecta al Departamento de Español de Tamkang, éste fomenta las representaciones teatrales, concursos de lectura, canciones, bailes, cuentacuentos, drama performance y cocina española. Los estudiantes también organizan exposiciones de libros de editoriales españolas, de artesanía, cerámica, escultura, procedentes de España y de los países latinoamericanos. Entre las actividades extraacadémicas, hay que destacar el «Congreso de Didáctica de Español» de carácter bienal, simposios y seminarios sobre el aprendizaje del español a los que suelen acudir profesores de universidades españolas e hispanoamericanas. Por último, Wenzao, que se centra en organizar un ciclo de conferencias sobre algún tema que interese al alumnado o al profesorado, por ejemplo, conferencias acerca de la traducción en el campo de la didáctica y el español con fines específicos. Hay también una Semana Cultural con distintas actividades para dar a conocer la cultura, el folklore y la gastronomía hispana; concursos de canciones, teatro, recitación, trabalenguas y baile. 
Un aspecto a destacar de los departamentos de español es el diseño y puesta en práctica de los programas de intercambio internacional. Las cuatro universidades tienen firmados convenios con universidades españolas e hispanoamericanas. El Departamento de Español de Fujen mantiene convenios en España con la Universidad Complutense de Madrid, Universidad de Alicante, Universidad de Salamanca, Universidad de Valladolid y la Universidad de Alcalá de Henares, y en cooperación con ellas envía, por un año académico, a una decena de alumnos. En los últimos años se ha estimulado a los estudiantes a participar, en verano, en cursos intensivos de español (corta duración) en la Universidad de Valladolid y Universidad de Alcalá de Henares. El Departamento de Español de Providence desde 1998 tiene un programa de intercambio con las Universidades de Salamanca y de Valladolid, desplazando a unos 30 estudiantes. También, existen planes de intercambio con la Universitat Autónoma de Barcelona, la de Alcalá de Henares y la de León, además de otras universidades hispanoamericanas. Por su parte Tamkang siguiendo la misma política, mantiene convenios con universidades españolas e hispanoamericanas, tanto para intercambios como para cursos intensivos durante el verano. Por último, es de señalar que el Departamento de Español de Wenzao, a través de sus convenios hispanoamericanos, mueve a más de 60 alumnos/as en los programas de intercambio internacionales y a ello hay que añadir becas para que estudien en el extranjero durante seis meses o un año.

\section{Los planes de estudio}

A la hora de analizar los planes de estudio nos hemos centrado en el curso académico 20I3-Ior4 y en las cuatro universidades que cuentan con departamento de español (Fujen, Providence, Tamkang y Wenzao). Tomamos como base de análisis los siguientes aspectos: tipo de asignatura, duración, crédito y asignaturas relacionadas con el español. El estudio de los planes de estudio se ha realizado departamento por departamento. Los planes de estudio de las cuatro universidades abarcan cuatro años y se organizan en torno a asignaturas obligatorias comunes, obligatorias de departamento y optativas. Suelen ser anuales o semestrales y con un valor entre de $2-8$ créditos.

\section{I. Universidad Católica de Funjen}

En el Departamento de Lengua de Funjen (ver Cuadro n. ${ }^{\circ}$ 2, las asignaturas optativas aparecen a pie de página) las materias de Formación Militar, Lengua Extranjera, Educación Física, Informática y Conocimientos Generales... son asignaturas obligatorias comunes para todas las facultades. La meta de estas asignaturas es conseguir el desarrollo integral, físico, social, intelectual, estético, moral y espiritual del alumnado. Se entiende que con estas materias se quiere conseguir formar a un ciudadano/a con unos valores determinados.

El Departamento de Español de Fujen distribuye en los dos primeros años las asignaturas relacionadas con la enseñanza en las cuatro capacidades lingüísticas: oír, hablar, leer y escribir. De esta forma, intenta motivar e interesar a los estudiantes en el conocimiento del español. En los dos últimos años, aparte de seguir consolidando los conocimientos de 
lengua, se presentan otras asignaturas con el fin de conseguir una sólida formación humanística además de la posibilidad de elegir de acuerdo con los intereses del alumnado.

\section{Cuadro 2: Asignaturas y créditos del Departamento de Español de la Universidad Católica de Fujen}

\begin{tabular}{|c|c|c|c|c|c|c|}
\hline \multicolumn{7}{|c|}{ Universidad Católica de Fujen: Departamento de Lenguas y Culturas Hispánicas } \\
\hline \multicolumn{7}{|c|}{ Curso académico 2013-2014 } \\
\hline Tipo & Asignatura & Duración & Tipo de curso & \multicolumn{3}{|c|}{ Crédito } \\
\hline \multicolumn{7}{|c|}{$1^{\circ} \mathrm{curso}$} \\
\hline \multirow{7}{*}{$\begin{array}{l}\text { Asignaturas } \\
\text { obligatorias } \\
\text { comunes }\end{array}$} & Tutoría & Anual & Obligatorio & 0 & 0 & 0 \\
\hline & Formación Militar & Anual & Obligatorio & 0 & 0 & 0 \\
\hline & Introducción a la Universidad & Semestral & Obligatorio & 2 & $\cdots$ & 2 \\
\hline & Educación Física & Anual & Obligatorio & 0 & 0 & 0 \\
\hline & $\begin{array}{l}\text { El ámbito de las Humanidades } \\
\text { y Artístico }\end{array}$ & Anual & Obligatorio & 2 & 2 & 4 \\
\hline & $\begin{array}{l}\text { El ámbito de las Ciencias } \\
\text { Naturales y Tecnología }\end{array}$ & Anual & Obligatorio & 2 & 2 & 4 \\
\hline & $\begin{array}{l}\text { El ámbito de las Ciencias } \\
\text { Sociales }\end{array}$ & Anual & Obligatorio & 2 & 2 & 4 \\
\hline \multirow{5}{*}{$\begin{array}{l}\text { Asignaturas } \\
\text { obligatorias } \\
\text { del } \\
\text { Departamento }\end{array}$} & Gramática I & Anual & Obligatorio & 2 & 2 & 4 \\
\hline & Conversación I & Anual & Obligatorio & 2 & 2 & 4 \\
\hline & Lectura I & Anual & Obligatorio & 2 & 2 & 4 \\
\hline & Escritura (I) & Anual & Obligatorio & 2 & 2 & 4 \\
\hline & Práctica Auditiva & Anual & Obligatorio & 2 & 2 & 4 \\
\hline \multicolumn{7}{|c|}{$2^{\circ} \mathrm{curso}$} \\
\hline \multirow{3}{*}{$\begin{array}{l}\text { Asignaturas } \\
\text { obligatorias } \\
\text { del } \\
\text { Departamento }\end{array}$} & Conversación II & Anual & Obligatorio & 4 & 4 & 8 \\
\hline & Lectura II & Anual & Obligatorio & 2 & 2 & 4 \\
\hline & Composición II & Anual & Obligatorio & 2 & 2 & 4 \\
\hline \multicolumn{7}{|c|}{$3^{\circ}$ curso } \\
\hline \multirow{2}{*}{$\begin{array}{l}\text { Asignaturas } \\
\text { obligatorias } \\
\text { comunes } \\
\end{array}$} & Tutoría & Anual & Obligatorio & 0 & 0 & 0 \\
\hline & Filosofía para la Vida & Anual & Obligatorio & 2 & 2 & 4 \\
\hline \multirow{5}{*}{$\begin{array}{l}\text { Asignaturas } \\
\text { obligatorias } \\
\text { del } \\
\text { Departamento }\end{array}$} & Comunicación Intercultural & Semestral & Obligatorio & 2 & ---- & 2 \\
\hline & Periodismo en Inglés & Semestral & Obligatorio & +-- & 2 & 2 \\
\hline & Gramática III & Anual & Obligatorio & 2 & 2 & 4 \\
\hline & Conversación III & Anual & Obligatorio & 2 & 2 & 4 \\
\hline & Lectura III & Anual & Obligatorio & 2 & 2 & 4 \\
\hline \multicolumn{7}{|c|}{$4^{\circ}$ curso } \\
\hline \multirow{2}{*}{$\begin{array}{l}\text { Asignaturas } \\
\text { obligatorias } \\
\text { comunes }\end{array}$} & Tutoría & Anual & Obligatorio & 0 & 0 & 0 \\
\hline & Ética Profesional & Semestral & Obligatorio & 2 & $-\cdots$ & 2 \\
\hline \multirow{3}{*}{$\begin{array}{l}\text { Asignaturas } \\
\text { obligatorias } \\
\text { del } \\
\text { Departamento }\end{array}$} & Gramática y Escritura (IV) & Anual & Obligatorio & 2 & 2 & 4 \\
\hline & Conversación (IV) & Anual & Obligatorio & 2 & 2 & 4 \\
\hline & $\begin{array}{l}\text { Comprensión Auditiva del } \\
\text { Inglés }\end{array}$ & Anual & Obligatorio & 2 & 2 & 4 \\
\hline
\end{tabular}


Las asignaturas obligatorias de español están dirigidas a la adquisición de las cuatro destrezas básicas del español (oír, hablar, leer, escribir). En cuanto a las asignaturas optativas existe una gran variedad ${ }^{4}$ para fomentar los conocimientos, las destrezas y las habilidades específicas del alumnado. Éstas favorecen el desarrollo de la competencia comunicativa intercultural, dicha competencia es potenciada desde los organismos internacionales (Marco Común Europeo de Referencia para las Lenguas y el Plan Curricular del Instituto Cervantes) por lo que consideramos que se deberían aumentar estas materias optativas tanto en número como en horas.

El plan de estudios de Fujen tiene un 53,I\% de asignaturas obligatorias de español, un $25 \%$ de obligatorias comunes y un 21,8\% de optativas de español. Así podemos constatar que algo más de la mitad de las materias son obligatorias de español (53,1\%), considerando esta proporción adecuada teniendo en cuenta la especialidad. Sin embargo, el porcentaje de las asignaturas optativas de español (2I,8\%) es algo bajo. Ante estos datos consideramos que sería necesario aumentar la proporción de las asignaturas optativas. También observamos déficit de asignaturas relacionadas con la didáctica de las lenguas y la didáctica de la lengua extranjera, aspecto que nos parece importante destacar ya que una salida profesional es la enseñanza del español.

\section{Cundro 3: Número de créditos por tipo de asignatura en el Departamento de Español de la Universidad Católica de Fujen}

\begin{tabular}{|c|c|c|}
\hline Tipo & $\mathrm{N}^{\circ}$ de créditos & Porcentaje \\
\hline General (Asignaturas obligatorias comunes) & 32 & $25 \%$ \\
\hline Disciplinar (Asignaturas obligatorias de español) & 68 & $53,1 \%$ \\
\hline Disciplinar y de profundización (Asignaturas optativas de español) & 28 & $21,8 \%$ \\
\hline Total del programa & 128 & $100 \%$ \\
\hline
\end{tabular}

Fuente: Huang (2015: 332).

\subsection{La Universidad de Providence}

La Universidad de Providence organiza la docencia en torno a asignaturas obligatorias comunes (generales), obligatorias de departamento y optativas. En el caso del

4 Asignaturas optativas obligatorias del Departamento de Español de Fujen: A) Segundo curso: Lectura de Textos Cortos Españoles, Lectura de Textos Cortos Latinoamericanos, Panorama del Mundo Hispánico (I), Panorama del Mundo Hispánico (II), Aplicación del Vocabulario Español (I), Aplicación del Vocabulario Español (II), Geografía y Cultura de España, Metodología en la Investigación Comparativa Transcultural, Biblia y Civilización.

B) Tercer curso: Literatura y Cine hispánicos, Correspondencia Comercial Española (I), Correspondencia Comercial Española (II), Traducción (Español-Chino), Lectores de Noticias y Aplicación del Vocabulario (I), Lectores de Noticias y Aplicación de Vocabulario (II), Gramática, oral y habilidades de escritura (I), Gramática, oral y habilidades de escritura (II), Multiculturalismo y su impacto social: Los inmigrantes latinos y asiáticos en EE. UU. (I), Multiculturalismo y su impacto social: Los inmigrantes latinos y asiáticos en EE. UU. (II), Intercultures and Interarts in Spanish, España y la Unión Europea, Hispanic News Transediting, Cultura china a través de las lenguas extranjeras-español (I), Cultura china a través de las lenguas extranjeras-español (II).

C) Cuarto curso: Introducción a la interpretación, Arte de Performance (I), Arte de Performance (II), Traducción (Chino-Español), Sociedad hispánica del siglo XXI (I), Sociedad hispánica del siglo XXI (II), Traducción y Comunicación en los negocios (I), Traducción y Comunicación en los negocios (II), Comunicación empresarial internacional (I), Comunicación empresarial internacional (II), Literatura en acción. 
Departamento de Español la estructura del plan de estudios se distribuye en torno a dos etapas. En la primera, nos encontramos con materias básicas (primer curso) y otras de cierta dificultad (segundo curso) con el objetivo de incrementar las habilidades básicas correspondientes a las destrezas: comprensión auditiva, expresión oral,

\section{CuAdro 4: Asignaturas y créditos del Departamento de Español de la Universidad} de Providence

\begin{tabular}{|c|c|c|c|c|c|c|}
\hline \multicolumn{7}{|c|}{ Universid ad de Providence: Departamento de Lengua y Literatura Española } \\
\hline \multicolumn{7}{|c|}{ Curso académico 2013-2014 } \\
\hline \multicolumn{7}{|c|}{$\begin{array}{l}\text { Créditos necesarios para graduarse: } 136 \text { créditos. Asignaturas obligatorias comunes (para todos los departamentos): } 33 \text { créditos. } \\
\text { Asignaturas obligatorias de español: } 66 \text { créditos. Asignaturas optativas de español: } 37 \text { créditos. }\end{array}$} \\
\hline \multirow[t]{2}{*}{ Tipo } & \multirow[t]{2}{*}{ Asignatura } & \multirow[t]{2}{*}{ Duración } & \multirow[t]{2}{*}{ Tipo de curso } & \multicolumn{3}{|c|}{ Crédito } \\
\hline & & & & $\begin{array}{l}1^{\circ} \\
\text { Semetre }\end{array}$ & $\begin{array}{l}2^{\mathrm{a}} \\
\text { Semes } \\
\text {-tre }\end{array}$ & $\begin{array}{l}\text { Total } \\
\text { crédito }\end{array}$ \\
\hline \multicolumn{7}{|c|}{$1^{\circ} \mathrm{curso}$} \\
\hline \multirow{10}{*}{$\begin{array}{l}\text { Asignaturas } \\
\text { obligatoria } \\
\text { comunes }\end{array}$} & Inglés (I) & Semestral & Obligatorio & 2 & $-\ldots$ & 2 \\
\hline & Inglés (II) & Semestral & Obligatorio & --- & 2 & 2 \\
\hline & Lectura y Escritura (I) & Semestral & Obligatorio & 2 & $-\ldots$ & 2 \\
\hline & Lectura y Escritura (II) & Semestral & Obligatorio & $\ldots$ & 2 & 2 \\
\hline & Educación Física (ejercicio y la salud) & Semestral & Obligatorio & 1 & ---- & 1 \\
\hline & Educación Física (deportes acuáticos) & Semestral & Obligatorio & 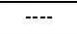 & 1 & 1 \\
\hline & Servicio-aprendizaje & Semestral & Obligatorio & & & 0 \\
\hline & Humanidades & Semestral & Obligatorio & & & 0 \\
\hline & Conocimientos generales & Semestral & Obligatorio & 2 & 2 & $\begin{array}{c}\text { Total: } \\
18 \\
\text { crédito }\end{array}$ \\
\hline & Introducción a la Informática & Semestral & Obligatorio & 3 & $-\cdots-$ & 3 \\
\hline \multirow{7}{*}{$\begin{array}{l}\text { Asignaturas } \\
\text { obligatorias } \\
\text { del } \\
\text { Departa- } \\
\text { mento }\end{array}$} & Self-Access Learning for FENM (I) (II) & Anual & Obligatorio & 0 & 0 & 0 \\
\hline & Gramática Española Nivel elemental (I) & Semestral & Obligatorio & 3 & 0 & 3 \\
\hline & Gramática Española Nivel elemental (II) & Semestral & Obligatorio & 0 & 3 & 3 \\
\hline & Conversación Española Nivel elemental (I) (+ prácticas) & Semestral & Obligatorio & 4 & 0 & 4 \\
\hline & Conversación Española Nivel elemental (II)(+ prácticas) & Semestral & Obligatorio & 0 & 4 & 4 \\
\hline & Comprensión lectora y escrita en Español Nivel elemental (I) & Semestral & Obligatorio & 2 & 0 & 2 \\
\hline & Comprensión lectora y escrita en Español Nivel elemental (II) & Semestral & Obligatorio & 0 & 2 & 2 \\
\hline \multicolumn{7}{|c|}{$22^{\circ}$ curso } \\
\hline \multirow{2}{*}{$\begin{array}{l}\text { Asignaturas } \\
\text { obligatorias } \\
\text { comunes }\end{array}$} & Educación Física I & Semestral & Obligatorio & 1 & 0 & 1 \\
\hline & Educación Física II & Semestral & Obligatorio & 0 & 1 & 1 \\
\hline \multirow{6}{*}{$\begin{array}{l}\text { Asignaturas } \\
\text { obligatorias } \\
\text { del } \\
\text { Departa- } \\
\text { mento }\end{array}$} & Gramática Española Nivel intermedia (I) & Semestral & Obligatorio & 2 & 0 & 2 \\
\hline & Gramática Española Nivel intermedia (II) & Semestral & Obligatorio & 0 & 2 & 2 \\
\hline & Conversación Española Nivel intermedia (+ prácticas) (I) & Semestral & Obligatorio & 4 & 0 & 4 \\
\hline & Conversación Española Nivel intermedia (+ prácticas) (II) & Semestral & Obligatorio & 0 & 4 & 4 \\
\hline & Comprensión lectora y escrita en Español Nivel intermedio (I) & Semestral & Obligatorio & 2 & 0 & 2 \\
\hline & Comprensión lectora y escrita en Español Nivel inter. (II) & Semestral & Obligatorio & 0 & 2 & 2 \\
\hline \multicolumn{7}{|c|}{ 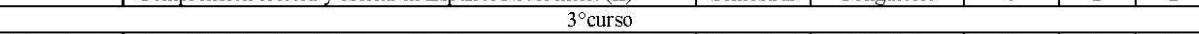 } \\
\hline \multirow{8}{*}{$\begin{array}{l}\text { Asignaturas } \\
\text { obligatorias } \\
\text { del } \\
\text { Departa- } \\
\text { mento }\end{array}$} & Gramática Española Nivel superior (I) & Semestral & Obligatorio & 2 & 0 & 2 \\
\hline & Gramática Española Nivel superior (II) & Semestral & Obligatorio & 0 & 2 & 2 \\
\hline & Conversación Española Nivel superior (I) & Semestral & Obligatorio & 4 & 0 & 4 \\
\hline & Conversación Española Nivel superior (II) & Semestral & Obligatorio & 0 & 4 & 4 \\
\hline & Comprensión lectora y escrita en Español Nivel superior (I) & Semestral & Obligatorio & 2 & 0 & 2 \\
\hline & Comprensión lectora y escrita en Español Nivel superior (II) & Semestral & Obligatorio & 0 & 2 & 2 \\
\hline & Traducción (Español- Chino) (I) & Semestral & Obligatorio & 2 & 0 & 2 \\
\hline & Traducción (Español- Chino) (II) & Semestral & Obligatorio & 0 & 2 & 2 \\
\hline \multicolumn{7}{|c|}{$4^{\circ}$ curso } \\
\hline \multirow{2}{*}{$\begin{array}{l}\text { Asignaturas } \\
\text { ob. Dep. }\end{array}$} & Conversación Española Nivel avanzado & Anual & Obligatorio & 4 & 4 & 8 \\
\hline & Traducción (Chino-Español) (I)(II) & Anual & Obligatorio & 2 & 2 & 4 \\
\hline
\end{tabular}


comprensión lectora y expresión escrita. En la segunda etapa (tercer y cuarto curso), hay varias asignaturas con matiz profesional como: Literatura hispánica, Cultura hispánica, Comercio, Turismo, Periodismo, Arte, etc., dirigidas hacia la especialidad.

Las asignaturas obligatorias del departamento de español (ver Cuadro n. ${ }^{\circ} 4$ ) son: Gramática (de I. ${ }^{\circ}$ a $4 .^{\circ}$ ), Conversación (de I. ${ }^{\circ}$ a $4 .^{\circ}$ ), Comprensión lectora y expresión

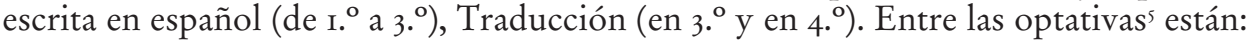
Introducción a la civilización hispánica e hispanoamericana, Introducción a la literatura hispánica, y en cuarto curso, destacar la Didáctica de la enseñanza del español como lengua extranjera: material y métodos, etc. La proporción de las asignaturas quedaría de la siguiente manera: el 48,5\% se corresponde con obligatorias de español, el $24,5 \%$, con asignaturas generales y el $27,2 \%$ con optativas de español.

En el plan de estudios de Providence hay que destacar la gran cantidad de asignaturas optativas que existen por curso, aspecto que no se da en todos los planes analizados y que creemos puede favorecer que los estudiantes elijan en función de sus preferencias. Estas asignaturas suelen ser más motivadoras, ya porque los profesores utilizan metodologías más activas, trabajan con grupos más reducidos o por realizar más actividades. Suelen ser más interesantes y favorecen el desarrollo de la competencia comunicativa intercultural del alumnado. Por último, señalar que la proporción de las asignaturas del Departamento de Español de Providence es adecuada.

\section{Cuadro 5: Número de créditos por tipo de asignatura en el Departamento de Español} de la Universidad de Providence

\begin{tabular}{|l|c|c|}
\hline \multicolumn{1}{|c|}{ Tipo } & $\mathrm{N}^{\circ}$ de créditos & Porcentaje \\
\hline General (Asignaturas obligatorias comunes) & 33 & $24,2 \%$ \\
\hline Disciplinar (Asignaturas obligatorias de español) & 66 & $48,5 \%$ \\
\hline Disciplinar y de profundización (Asignaturas optativas de español) & 37 & $27,2 \%$ \\
\hline Total del programa & 136 & $100 \%$ \\
\hline
\end{tabular}

Fuente: Huang (2015: 338).

\subsection{La Universidad de Tamkang}

La tercera Universidad en la que se centra nuestro estudio es la de Tamkang. Su Departamento de Español organiza el plan de estudios en asignaturas obligatorias

Asignaturas optativas del Departamento de Español de la Universidad de Providence: A) Primer curso: Introducción a la civilización hispánica, Introducción a la civilización hispanoamericana, Práctica de Frases Fundamental (I), Práctica de Frases Fundamental (II), Vocabulario y Uso (I), Vocabulario y Uso (II), ALL-OU defensa de educación militar de entrenamiento (I IV).

B) Segundo curso: Comprensión Audio-visual (I), Comprensión Audio-visual (II), Práctica de Sintaxis Española (I), Práctica de Sintaxis Española (II), Arte Española (I), Arte Española (II), Introducción a la literatura hispánica (I), Introducción a la literatura hispánica (II).

C) Tercer curso: Vida y cultura de España (I), Vida y cultura de España (II), Periodismo Español (I), Periodismo Español (II), Español para el turismo (I), Español para el turismo (II), Introducción a la Lingüística Española (I), Introducción a la Lingüística Española (II), Historia contemporánea de los países hispanoamericanos (I), Historia contemporánea de los países hispanoamericanos (II), Español para los $\mathrm{Ne}$ gocios y Administración (I), Español para los Negocios y Administración (II), Literatura hispana: Lectura y apreciación (I), Literatura hispana: Lectura y apreciación (II).

D) Cuarto curso: Traducción del Periodismo Español, Correspondencia Comercial en Español, Crítica literaria, Español Nivel avanzado (I) (II), Historia y cultura de Latinoamérica, Música de los países de habla hispana, Toda la formación en lengua española, Didáctica de la enseñanza del español como lengua extranjera: material y métodos, Práctica de la enseñanza de español como lengua extranjera. 
comunes, obligatorias de departamento y optativas ${ }^{6}$, al igual que las otras dos universidades. En este caso, en los dos primeros años, se da importancia a las clases de gramática, conversación, lectura, composición, historia, geografía y audiovisuales, con el objetivo de desarrollar las cuatro habilidades esenciales. En los dos años siguientes se hace más hincapié en las clases de gramática, conversación y lectura con un nivel más avanzado. También hay asignaturas más específicas: Literatura Española e Hispanoamericana, Economía, Teatro, Traducción, Cultura, Arte y Turismo, etc., dentro de la optatividad que se ofrece en primer, segundo, tercer y cuarto año de carrera.

Cuadro 6: Número de créditos por tipo de asignatura en el Departamento de Español de la Universidad de Tamkang

\begin{tabular}{|l|c|c|}
\hline \multicolumn{1}{|c|}{ Tipo } & $\mathrm{N}^{\circ}$ de créditos & Porcentaje \\
\hline General (Asignaturas obligatorias comunes) & 31 & $22,7 \%$ \\
\hline Disciplinar (Asignaturas obligatorias de español) & 72 & $52,9 \%$ \\
\hline Disciplinar y de profundización (Asignaturas optativas de español) & 21 & $15,4 \%$ \\
\hline Total del programa & 136 & $100 \%$ \\
\hline
\end{tabular}

Fuente: Huang (2015: 343).

Según se observa en el cuadro anterior el 52,9\% de las asignaturas se corresponden con obligatorias de español: Gramática $\left(\right.$ de $~ .^{\circ}$ a $\left.4 .^{\circ}\right)$, Conversación (de r. ${ }^{\circ}$ a $\left.4 .^{\circ}\right)$, Práctica auditiva (en $\mathrm{r} .{ }^{\circ}$ y en $2 .^{\circ}$ ), Composición (en $2 .^{\circ}$ y en $3 .^{\circ}$ ), Interpretación y Traducción oral (en $3 .^{\circ}$ y en $4 .^{\circ}$ ) e Introducción de la literatura española/ latinoamericana (en $3 .^{\circ}$ ). A éstas hay que añadir algunas optativas como Introducción a la lingüística española, Historia y Geografía de Hispanoamérica, Español aplicado a la enseñanza del chino y Arte Hispánico, etc., que completan la formación desde una perspectiva sociolingüística. Por lo que se refiere al porcentaje de las asignaturas obligatorias comunes (generales), éste supone el $22,7 \%$ y el I5,4\% se correspondería con las optativas de español. Ante esta situación podemos pensar que los créditos de las optativas de español son escasos. Así pues, es necesario reajustar la proporción de los créditos para ofrecer a los estudiantes más asignaturas optativas de español.

\subsection{Universidad de Idiomas Extranjeros Wenzao}

Por último, presentamos el plan de estudios del Departamento de Español de la Universidad de Idiomas Extranjeros Wenzao, en su modalidad Grado de cuatro años. El plan de estudios está diseñado bajo una conceptualización global, poniendo énfasis en los valores de la cultura china, de las ciencias y de las humanidades con el objetivo de que los estudiantes adquieran una visión amplia del mundo. Para ello se han

6 Asignaturas optativas del Departamento de Español de Tamkang: A) Primer curso: Práctica Auditiva I (+ prácticas), Historia y Geografía de Hispanoamérica.

B) Segundo curso: Léxico español y lectura (I), Práctica Auditiva II (+ prácticas), Introducción a la lingüística española.

C) Tercer curso: Léxico español y lectura (II), Español para el Turismo, Cultura Española, Español aplicado a la enseñanza del chino.

D) Cuarto curso: Gramática Española Nivel superior, Español aplicado a la enseñanza del chino, Español aplicado, Arte Hispánico, Obras maestras de la novela española, Obras maestras del teatro español, Tutoriales de Español. 
Cuadro 7: Asignaturas y créditos del Departamento de Español de la Universidad de Tamkang

\begin{tabular}{|c|c|c|c|c|c|c|}
\hline \multicolumn{7}{|c|}{ Universidad de Tamkang: Departamento de Español } \\
\hline \multicolumn{7}{|c|}{ Curso académico 2013-2014 } \\
\hline \multicolumn{7}{|c|}{$\begin{array}{l}\text { Créditos necesarios para graduarse: } 136 \text { créditos. Asignaturas obligatorias comunes (para todos los departamentos): } 31 \text { créditos. } \\
\text { Asignaturas obligatorias de español: } 72 \text { créditos. Asignaturas optativas de español: } 21 \text { créditos. }\end{array}$} \\
\hline \multirow[t]{2}{*}{ Tipo } & \multirow[t]{2}{*}{ Asignatura } & \multirow[t]{2}{*}{ Duración } & \multirow[t]{2}{*}{ Tipo de curso } & \multicolumn{3}{|c|}{ Crédito } \\
\hline & & & & $\begin{array}{c}1^{\mathrm{s}} \\
\text { Semes } \\
\text {-tre }\end{array}$ & $\begin{array}{c}2^{\mathrm{a}} \\
\text { Semes } \\
\text { tre }\end{array}$ & T crédito \\
\hline \multicolumn{7}{|c|}{$1^{\circ}$ curso } \\
\hline \multirow{15}{*}{$\begin{array}{l}\text { Asignaturas } \\
\text { obligatorias } \\
\text { comunes }\end{array}$} & Inglẻs (I) & Anual & Obligatorio & 2 & 2 & 4 \\
\hline & Educación Física & Anual & Obligatorio & 0 & 0 & 0 \\
\hline & Introducción a la Informática & Anual & Obligatorio & 2 & 2 & 4 \\
\hline & Revolución tecnológica global & Semestral & Obligatorio & 2 & $-\ldots$ & 2 \\
\hline & Servicio-Aprendizaje & Anual & Obligatorio & 0 & 0 & 0 \\
\hline & Entrenamiento militar/Enfermería & Anual & Obligatorio & 0 & 0 & 0 \\
\hline & Examen de inglés & Semestral & Obligatorio & $-\ldots$ & 0 & 0 \\
\hline & Conocimientos generales (Literatura y texto clásicos) ${ }^{*}$ & Semestral & Obligatorio & 2 & 2 & 4 \\
\hline & Conocimientos generales (Historia y cultura) * & Semestral & Obligatorio & 2 & 2 & 4 \\
\hline & Conocimientos generales (Filosofia y Religión) * & Semestral & Obligatorio & 2 & 2 & 4 \\
\hline & El ámbito de desarrollo social * & Semestral & Obligatorio & 2 & 2 & $\begin{array}{l}4^{*} \text { Tota } \\
1: 8 \\
\text { crédito }\end{array}$ \\
\hline & Conocimientos generales (Apren dizaje y desarrollo) \# & Semestral & Obligatorio & 2 & 2 & 4 \\
\hline & Conocimientos generales (Futurología) \# & Semestral & Obligatorio & 2 & 2 & 4 \\
\hline & Conocimientos generales (Perspectiva global) \# & Semestral & Obligatorio & 2 & 2 & $\begin{array}{l}4 \# \\
\text { Total: } \\
6 \\
\text { crédito }\end{array}$ \\
\hline & Actividades Extracurriculares y Desarrollo de Equipos & Semestral & Obligatorio & $-\cdots-$ & 1 & 1 \\
\hline \multirow{3}{*}{$\begin{array}{l}\text { Asignaturas } \\
\text { obligatorias } \\
\text { del Dpto. }\end{array}$} & Gramática Española Nivel elemental & Anual & Obligatorio & 4 & 4 & 8 \\
\hline & Conversación & Anual & Obligatorio & 4 & 4 & 8 \\
\hline & Práctica de sintaxis española & Anual & Obligatorio & 2 & 2 & 4 \\
\hline \multicolumn{7}{|c|}{$2^{\circ}$ curso } \\
\hline \multirow{3}{*}{$\begin{array}{l}\text { Asignaturas } \\
\text { obligatorias } \\
\text { comunes }\end{array}$} & Capacidad de expresión en chino & Semestral & Obligatorio & $-\cdots-\cdot$ & 3 & 3 \\
\hline & Inglés (I) & Anual & Obligatorio & 2 & 2 & 4 \\
\hline & Educación Física & Anual & Obligatorio & 0 & $\overline{0}$ & 0 \\
\hline \multirow{3}{*}{$\begin{array}{l}\text { Asig. Ob. } \\
\text { Dpto. }\end{array}$} & Gramatica- Española Nivel intermedia & Anual & Obligatorio & 4 & 4 & 8 \\
\hline & Conversación (II) & Anual & Obligatorio & 4 & 4 & 8 \\
\hline & Composición (I) & Anual & Obligatorio & 2 & 2 & 4 \\
\hline \multicolumn{7}{|c|}{$3^{\circ}$ curso } \\
\hline Asig. Ob. Co. & Educación Física & Anual & Obligatorio & 0 & 0 & 0 \\
\hline \multirow{6}{*}{$\begin{array}{l}\text { Asignatura } \\
\text { Obl. } \\
\text { de } \\
\text { Depto. }\end{array}$} & Gramatica- Española Nivel avanzado & Anual & Obligatorio & 2 & 2 & 4 \\
\hline & Conversación (III) & Anual & Obligatorio & 4 & 4 & 8 \\
\hline & Composición (II) & Anual & Obligatorio & 2 & 2 & 4 \\
\hline & Interpretación-traducción oral (I) & Anual & Obligatorio & 2 & 2 & 4 \\
\hline & Introducción a la literatura española & Semestral & Obligatorio & 2 & $-\cdots$ & 2 \\
\hline & Introducción literatura latinoamericana & Semestral & Obligatorio & --- & 2 & 2 \\
\hline \multicolumn{7}{|c|}{$4^{\circ} \mathrm{curso}$} \\
\hline \multirow{2}{*}{$\begin{array}{l}\text { Asig. Ob. } \\
\text { Dpto. }\end{array}$} & Conversación (IV) & Anual & Obligatorio & 2 & 2 & 4 \\
\hline & Interpretación y traducción oral (II) & Anual & Obligatorio & 2 & 2 & 4 \\
\hline
\end{tabular}

Fuente: Huang (2015: 341-343).

seleccionado asignaturas relacionadas con el comercio internacional, con la evolución económica y social y con las tendencias del mercado laboral.

En los dos primeros años se dan clases de Gramática, Conversación, Composición, Lectura, Historia, Geografía y Audiovisuales, de nivel básico para conseguir las cuatro destrezas de la lengua: comprensión auditiva, comprensión escrita, expresión oral y expresión escrita. En los siguientes años, los estudiantes siguen teniendo clases de Gramática, Conversación, Composición y Lectura, pero a un nivel más avanzado 
LOS PLANES DE ESTUDIO DE ESPAÑOL EN LAS UNIVERSIDADES DE TAIWÁN

Cuadro 8: Asignaturas y créditos del Departamento de Español de la Universidad de Idiomas Extranjeros Wenzao: Grado de cuatro años

\begin{tabular}{|c|c|c|c|c|c|c|}
\hline \multicolumn{7}{|c|}{ Universidad de Idiomas Extranjeros Wenzao: Departamento de Español (cuatro anos) } \\
\hline \multicolumn{7}{|c|}{ Curso académico 2013-2014 } \\
\hline \multicolumn{7}{|c|}{$\begin{array}{l}\text { Créditos necesarios para graduarse: } 145 \text { créditos. Asignaturas obligatorias comunes (para todos los departamentos): } 49 \mathrm{créditos} \text {. } \\
\text { Asignaturas obligatorias de español: } 64 \text { créditos. Asignaturas optativas de español: } 8 \text { créditos. Asignaturas interdisciplinarias } \\
\text { especializadas en materias españolas: } 20 \text { créditos. }\end{array}$} \\
\hline \multirow{2}{*}{\multicolumn{2}{|c|}{ Asignatura }} & \multirow[t]{2}{*}{ Duración } & \multirow[t]{2}{*}{ Tipo de curso } & \multicolumn{3}{|c|}{ Crédito } \\
\hline & & & & $1^{\circ}$ Semestre & $2^{\circ}$ Semestre & T.crédito \\
\hline \multicolumn{7}{|c|}{$1^{\circ}$ curso } \\
\hline \multirow{11}{*}{$\begin{array}{l}\text { Asignaturas } \\
\text { obligatorias } \\
\text { comunes }\end{array}$} & Inglés I & Anual & Obligatorio & 4 & 4 & 4 \\
\hline & Introducción a la Informática & Semestral & Obligatorio & 2 & ---- & 2 \\
\hline & Introducción a la multimedia & Semestral & Obligatorio & ---- & 2 & 2 \\
\hline & $\begin{array}{l}\text { Conocimientos gen erales(sociedad y } \\
\text { leyes) }\end{array}$ & Semestral & Obligatorio & 2 & $+--\cdot$ & 2 \\
\hline & Conocimientos generales (Lógica) & Semestral & Obligatorio & $-\cdots$ & 2 & 2 \\
\hline & Introducción a la Universidad (I) & Semestral & Obligatorio & 0 & $\overline{c---}$ & 0 \\
\hline & Introducción a la Universidad (II) & Semestral & Obligatorio & $\cdots$ & 0 & 0 \\
\hline & Educación Física & Anual & Obligatorio & 0 & 0 & 0 \\
\hline & Servicio-aprendizaje & Semestral & Obligatorio & $-\cdots$ & 1 & 1 \\
\hline & Literatura china moderna & Semestral & Obligatorio & 2 & 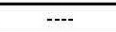 & 2 \\
\hline & Introducción al Arte chino & Semestral & Obligatorio & +-- & 2 & 2 \\
\hline \multirow{3}{*}{$\begin{array}{l}\text { Asignaturas } \\
\text { obligatorias } \\
\text { del } \\
\text { Departamento }\end{array}$} & Espafiol I & Anual & Obligatorio & 4 & 4 & 8 \\
\hline & Conversación I & Anual & Obligatorio & 4 & 4 & 8 \\
\hline & Lectura y audición I & Anual & Obligatorio & 2 & 2 & 4 \\
\hline \multicolumn{7}{|c|}{$2^{\circ}$ curso } \\
\hline \multirow{7}{*}{$\begin{array}{l}\text { Asignaturas } \\
\text { obligatorias } \\
\text { comunes }\end{array}$} & Inglès II & Anual & Obligatorio & 4 & 4 & 4 \\
\hline & $\begin{array}{l}\text { Conocimientos generales (cultura y } \\
\text { arte) }\end{array}$ & Semestral & Obligatorio & 2 & --- & 2 \\
\hline & $\begin{array}{l}\text { Conocimientos gen erales } \\
\text { (Naturaleza y su protección) }\end{array}$ & Semestral & Obligatorio & $-\cdots$ & 2 & 2 \\
\hline & Ética II & Semestral & Obligatorio & 0 & --- & 0 \\
\hline & Antología de la literatura clásica & Anual & Obligatorio & 2 & 2 & 2 \\
\hline & Chino aplicado & Semestral & Obligatorio & 0 & 2 & 2 \\
\hline & $\begin{array}{l}\text { Introducción a la comunicación } \\
\text { intercultural }\end{array}$ & Anual & Obligatorio & 2 & 2 & 2 \\
\hline \multirow{4}{*}{$\begin{array}{l}\text { Asignaturas } \\
\text { obligatorias } \\
\text { del } \\
\text { Departamento }\end{array}$} & Español II & Anual & Obligatorio & 4 & 4 & 8 \\
\hline & Conversación II & Anual & Obligatorio & 2 & 2 & 4 \\
\hline & Lectura y audición II & Anual & Obligatorio & 2 & 2 & 4 \\
\hline & Escritura I & Anual & Obligatorio & 2 & 2 & 4 \\
\hline \multicolumn{7}{|c|}{$3^{\circ} \mathrm{curso}$} \\
\hline \multirow{2}{*}{$\begin{array}{l}\text { Asignaturas } \\
\text { obligatorias } \\
\text { comunes }\end{array}$} & Inglés III & Anual & Obligatorio & 4 & 4 & 4 \\
\hline & Conocimientos generales & Semestral & Obligatorio & 2 & $-\cdots$ & 2 \\
\hline \multirow{4}{*}{$\begin{array}{l}\text { Asignaturas } \\
\text { obligatorias } \\
\text { del } \\
\text { Departamento }\end{array}$} & Espaniol III & Anual & Obligatorio & 2 & 2 & 4 \\
\hline & Conversación III & Anual & Obligatorio & 2 & 2 & 4 \\
\hline & Escritura II & Anual & Obligatorio & 2 & 2 & 4 \\
\hline & Historia y cultura espaniola & Semestral & Obligatorio & $-\cdots$ & 2 & 2 \\
\hline \multicolumn{7}{|c|}{$4^{\circ} \mathrm{curso}$} \\
\hline \multirow{4}{*}{$\begin{array}{l}\text { Asignaturas } \\
\text { obligatorias } \\
\text { del } \\
\text { Departamento }\end{array}$} & Español IV & Anual & Obligatorio & 2 & 2 & 4 \\
\hline & Interpretación & Anual & Obligatorio & 2 & 2 & 4 \\
\hline & Proyecto de Graduación & Anual & Obligatorio & 2 & 2 & 4 \\
\hline & Historia y cultura latinoamericana & Semestral & Obligatorio & $+\cdots$ & 2 & 2 \\
\hline \multirow{6}{*}{$\begin{array}{l}\text { Asignaturas } \\
\text { inter- } \\
\text { disciplinarias }\end{array}$} & Comercio intemacional & Anual & Optativo & 203 & 203 & 608 \\
\hline & Asuntos internacionales & Anual & Optativo & 3 & 3 & 6 \\
\hline & Difusión digital & Anual & Optativo & 3 & 3 & 6 \\
\hline & Enseñanza del chino & Anual & Optativo & 2 & 2 & 4 \\
\hline & Traducción & Anual & Optativo & 2 & 3 & 40506 \\
\hline & Turismo y culturas extranjeras & Anual & Optativo & 2 & 2 & $\begin{array}{c}4 \\
\text { T. } 20 \text { cre. }\end{array}$ \\
\hline
\end{tabular}


para consolidar las cuatro destrezas aprendidas. Además, con el objetivo de tener una formación más específica se cursan estudios de Traducción español-chino, Traducción chino-español, Literatura española o Literatura Latinoamericana, Teatro, Novela, Periodismo, Cultura y Arte, Turismo, Comercio, y Prácticas laborales, etc.

En el cuadro n. ${ }^{\circ} 9$ se observa que las asignaturas generales suman en $33,7 \%$, mientras que las obligatorias de español se mantienen en un $44, \mathrm{r} \%$; dejando el $5,5 \%$ y el $7,25 \%$ respectivamente, a las asignaturas optativas de español ${ }^{7}$ y las interdisciplinarias. Esto nos indica que el porcentaje de créditos de las asignaturas optativas de español y las interdisciplinarias es muy pequeño. Por el contrario, hay muchas asignaturas obligatorias comunes y el porcentaje entre éstas y las específicas de español es muy pequeño. Así pues, debería disminuir el porcentaje de las asignaturas generales para aumentar los créditos de las asignaturas específicas de español, optativas de español y las interdisciplinarias, potenciando un plan de estudios más actual en relación con las necesidades del Grado. Destacar en la optatividad la didáctica del español, pues hemos observado que no se da en todos los planes de estudio y creemos que es muy necesaria para entender y poder hablar una lengua.

Cuadro 9: Número de créditos por tipo de asignatura en el Departamento de Español de la Universidad de Idiomas Extranjeros Wenzao: Grado de cuatro años

\begin{tabular}{|l|c|c|}
\hline Área & $\mathrm{N}^{\circ}$ de créditos & Porcentaje \\
\hline General (Asignaturas obligatorias comunes) & 49 & $33,7 \%$ \\
\hline Disciplinar (Asignaturas obligatorias de español) & 64 & $44,1 \%$ \\
\hline Disciplinar y de profundización (Asignaturas optativas de español) & 8 & $5,5 \%$ \\
\hline Interdisciplinar (Asignaturas interdisciplinarias especializadas en materias españolas) & 20 & $7,25 \%$ \\
\hline Total del programa & 145 & $100 \%$ \\
\hline
\end{tabular}

Fuente: Huang (2015:352).

Como conclusiones extraídas de los planes de estudio podemos decir que, aunque se observan diferencias en algunos aspectos, también se dan coincidencias: dividir las asignaturas en comunes, específicas del español y optativas. Entre las primeras están: Chino mandarín, Inglés, Informática, Entrenamiento militar, Conocimientos generales, Educación Física... que tienen como objetivo proporcionar a los estudiantes conocimientos generales que puedan ayudarles tanto en el estudio de la especialidad, como en su futuro profesional. Pero también hay que reconocer, en algunas de ellas, cierto nivel de adoctrinamiento, desde una perspectiva occidental. Estas asignaturas suponen aproximadamente una cuarta parte del total. Las segundas, las asignaturas específicas de español, suman generalmente dos tercios en el horario total y se dividen en dos niveles: a) dos años de iniciación para que los estudiantes adquieran las destrezas básicas del español y b) otros dos de especialización para profundizar tanto en el idioma español como en la cultura hispánica. Estas asignaturas se pueden dividir en dos bloques (prácticas y teóricas) y en cuatro ámbitos (Práctica de lengua, Aplicación de lengua, Bloque cultura -suma de asignaturas socioculturales- y Literatura).

Asignaturas optativas del Departamento de Español de Wenzao grado 4 años: A) Tercer curso: Español para el turismo, Comercio.

B) Cuarto curso: Periodismo, Literatura española, Literatura latinoamericana, Introducción a la didáctica del español, Proyección y comentario de películas hispanoamericanas, Prácticas laborales en el extranjero, Práctica laboral (de poca duración), Prácticas laborales semestrales.

Como asignaturas interdisciplinares de cuarto curso están: Comercio internacional, Asuntos internacionales, Difusión digital, Enseñanza del chino, Traducción, Turismo y culturas extranjeras. 
Las asignaturas prácticas (español básico, español medio, español superior, español avanzado, conversación, audiovisual, lectura, composición, etc.) tienen como fin que los estudiantes adquieran las cuatro destrezas de la lengua: comprensión auditiva, comprensión lectora, expresión oral y expresión escrita, y se cursan progresivamente a lo largo de los cuatro años. Las asignaturas teóricas se imparten a partir del tercer y cuarto curso, con el propósito de profundizar en el conocimiento de la filología hispánica y de otros aspectos socioculturales del mundo hispanohablante. Se estudia lingüística, fonética y fonología, gramática, lexicología, introducción a la cultura española e hispanoamericana, literatura española e hispanoamericana, correspondencia comercial y relaciones internacionales de los países hispanohablantes entre otras.

En los dos años siguientes, se pone más énfasis en las clases de gramática, conversación y lectura con un nivel más avanzado para alcanzar las cuatro habilidades esenciales. Con el objetivo de tener una formación específica de español se ofrecen materias más específicas, como Traducción, Literatura, Teatro, Novela, Cultura y Arte, Español aplicado al periodismo, Español aplicado al turismo, Español comercial, etc. Generalmente, a partir del tercer curso, aparte de seguir afianzando los conocimientos de lengua, existen otras asignaturas para conseguir una formación humanística. Los estudiantes pueden elegir según sus intereses.

Podemos concluir que los planes de estudio de español de los cuatro Departamentos coinciden en integrar la lengua entre las cuatro destrezas básicas y mantener las asignaturas de Gramática, Lectura y Conversación desde I. ${ }^{\text {er }}$ curso hasta $3 .{ }^{\text {er }}$ curso (o hasta $4 .^{\circ}$ curso). Por lo que respecta a las asignaturas optativas, se ofrece una gran variedad de opciones: Literatura, Comercio, Turismo, Periodismo, Cultura, etc. Por otra parte, se observa un alto nivel de coincidencia entre las asignaturas obligatorias y optativas en los cuatro Departamentos de Español. El currículo de las cuatro universidades es muy parecido y se detecta escasez de materias relacionadas con la didáctica, tanto de la lengua como de la lengua extranjera.

\section{Conclusiones finales y propuestas}

En la actualidad existen en Taiwán cuatro universidades con un departamento de español: Católica de Fujen, Providence, Tamkang y la Universidad de Idiomas Extranjeros Wenzao, que expiden el título de Licenciatura en Lengua Española, con cuatro años de duración. El alumnado que llega a estos estudios, en su mayor parte, no posee conocimientos de español y a lo largo de los cuatro años estudia desde la gramática básica, la cultura, la historia, la literatura, el comercio, etc., hasta todo tipo de materias profesionales, para conocer la cultura meta. Estas universidades ofrecen también planes de intercambio y estancias en universidades hermanadas de España o Iberoamérica.

A través del análisis realizado sobre los planes de estudio de los cuatro Departamentos de Español, hemos observado algunas coincidencias, por lo que respecta a su diseño: integrar la lengua en las cuatro destrezas básicas; mantener una secuenciación en las asignaturas de Gramática, Lectura y Conversación desde I. ${ }^{\circ}$ hasta $3 .^{\circ}$ (o hasta $\left.4 .^{\circ}\right)$; tener asignaturas troncales muy parecidas, y presentar una gran variedad de asignaturas optativas. Los planes de estudio se estructuran en torno a asignaturas comunes para todos los estudiantes y específicas solo para los estudiantes de español. Las 
asignaturas comunes (Chino mandarín, Inglés, Informática, Entrenamiento militar, Conocimientos generales, Educación Física, etc.) tienen como objetivo formar a los estudiantes para que adquieran conocimientos generales (en ocasiones con gran carga patriótica) y representan aproximadamente una cuarta parte del total.

Como conclusión, podemos afirmar que los planes de estudio de las cuatro universidades tienen una orientación estructuralista (Cortés, 20I2: 84), centrada en la competencia lingüística en la que «La lengua es concebida como un conjunto de conocimientos - mayormente, normas y estructuras gramaticales, así como listas de léxico- que se transmiten de los libros y del docente a los estudiantes». Es decir, el sistema de enseñanza del español en Taiwán se centra más en la teoría que en la práctica. En Taiwán se da mucha importancia y se dedica mucho tiempo a la sistematicidad del método tradicional, pues solo cuando el estudiante ha interiorizado la gramática, practicado la fonética y memorizado largas listas de vocabulario, es cuando se considera que está preparado para hablar y participar en una conversación. Se trabajan las destrezas básicas de forma aislada, sin establecer relaciones entre ellas o entre el contexto sociocultural (Sánchez Griñán, 2006).

Estos departamentos no están en la avanzadilla metodológica en cuanto a la conceptualización de lo que debe ser el fin del proceso de enseñanza-aprendizaje del E/LE. En el siglo xxI, muchos expertos e instituciones internacionales han abogado por una lingüística aplicada al E/LE enfocada hacia el desarrollo de la competencia comunicativa. Tanto el Marco Común Europeo de Referencia para las Lenguas (MCER) (Consejo de Europa, 200I) como el Plan Curricular del Instituto Cervantes (PCIC) (2006) han propuesto nuevos enfoques centrados en el análisis de la lengua desde la perspectiva de la comunicación, la teoría del aprendizaje y la teoría de la educación. Así se señala la importancia de la relación entre la lengua y el contexto sociocultural, y la necesidad de integrar la competencia sociocultural y la competencia intercultural en los procesos de enseñanza-aprendizaje. Todo ello supone no solo prestar atención a las formas lingüísticas, sino a quienes las usan en situaciones de comunicación. De hecho, somos conscientes de que la competencia comunicativa va más allá de la mera competencia gramatical, léxica, fónica, etc., y que el componente pragmático e incluso el no verbal son determinantes en la comunicación humana. Cortés (2012: 88) afirma que:

Bajo la concepción actual, la lengua extranjera es un sistema de comunicación que cada aprendiente -agente social y hablante intercultural- construye interactuando con otros usuarios, formulando hipótesis y poniéndolas a prueba. El significado de cada enunciado no es del todo predecible a partir del libro de gramática ni del diccionario; se negocia durante el acto de comunicación y, en última instancia, depende del contexto lingüístico, de la situación de comunicación, de la relación entre los interlocutores y sus experiencias anteriores; en definitiva, depende, en buena medida, de la pragmática, una disciplina todavía ausente en el currículo.

Otra de las conclusiones a las que hemos llegado está relacionada con la competencia sociocultural e intercultural. Para poder conocer su importancia en los planes de estudio de E/LE (ante la gran variedad de asignaturas), hemos elaborado el concepto «bloque cultura», que engloba las materias que tienen como fin llegar a entender las realidades del entorno social de los países de habla hispánica, y ampliar el horizonte mental, cultural y humano del estudiante. Se busca, en definitiva, que sirva al alumnado de puente entre la cultura taiwanesa y la española. Pero los Departamentos de 
Español, tal y como se ha visto en los planes de estudio, se centran en la Literatura, Historia, Arte, Geografía, es decir, en los contenidos de "Cultura con mayúsculas». Estos temas tienden a ser exhaustivos, pero no se orientan hacia las necesidades del alumnado. La asignatura bloque cultura, para conseguir su objetivo, tendría que tomar en cuenta las recomendaciones del MCER y el PCIC y, solo así se podría adquirir la competencia comunicativa intercultural. Y para ello es necesario combinar los conocimientos socioculturales con destrezas y habilidades (sensibilidad cultural, pensamiento independiente, flexibilidad, actitud abierta de respecto y aceptación, etc.).

La falta de contenidos socioculturales de los planes de estudio conlleva ciertos problemas de comprensión respecto de la cultura meta. Los conocimientos culturales se centran en la "Cultura con mayúscula» como ya hemos señalado y ésta no guarda mucha relación con la vida cotidiana, por lo tanto, los estudiantes no pueden comprenderla fácilmente. Hay que potenciar los aspectos relacionados con la «Cultura con minúscula» y la «Kultura con k», que giran en torno a los aspectos socioculturales de la cultura meta, aspectos que potenciarían la competencia intercultural y la competencia comunicativa.

Vielva y Salas (2000: 32 ) destacan la importancia de los aspectos sociales en el lenguaje, siguiendo los principios de Vygotsky, al afirmar que

... el lenguaje es el instrumento de mediación semiótica que juega un papel decisivo en el proceso de interiorización. Son los signos y los símbolos las herramientas culturales que amarran o integran al individuo a la sociedad, y el principal mecanismo de esta unión lo constituyen el lenguaje y otras propiedades simbólicas.

Así pues los enfoques socioculturales (Vygotsky, 20Io) que nos ayudan a comprender la cultura y la influencia del contexto, en nuestro caso, el proceso de enseñanzaaprendizaje, deberían ser aspectos relevantes a la hora de enseñanza y poder comprender la cultura meta.

Avanzando en la idea de conocer mejor la cultura meta desde la propia cultura, hemos observado que en los planes de estudio no existen materias ni contenidos ni actividades relacionadas con la comunicación no verbal. La comunicación no verbal es un aspecto de gran relevancia en el aprendizaje de E/LE (ver Mehrabian, 1972), sobre todo en la cultura latina, llena de gestos y mímica. Por lo que respecta a la cultura china, la comunicación no verbal no es tan evidente, no se manifiesta de manera tan expresiva como en la española. Esto nos lleva a reflexionar sobre la importancia de incorporar en los estudios de E/LE todo aquello que tiene que ver con los gestos y expresiones no verbales, que condicionan la comprensión de la cultura meta. Por otra parte, si queremos que los estudiantes adquieran una competencia comunicativa plena y sean capaces de comunicarse con personas de otra cultura, tenemos que llevar a cabo un tipo de enseñanza-aprendizaje intercultural que incluya, también, el tratamiento de la comunicación no verbal.

Como consecuencia de lo analizado podemos pensar que la metodología que se utiliza en los departamentos de español es una combinación del método de Gramática-Traducción con el método Audiolingual, ya que está fuertemente influida por la propia tradición de la enseñanza china, en donde la repetición y la memorización tienen un papel muy importante. Las metodologías que se utilizan actualmente dependen mucho de la formación específica de cada docente. También hay que señalar la ausencia en los planes de estudio de materias relacionadas con la didáctica de la lengua 
y de la lengua extranjera; la rigidez que se observa en los programas, o el hecho de que las funciones comunicativas queden supeditadas a la gramática. Todos estos aspectos deberían estar presentes para que el alumnado pudiera adquirir los conocimientos declarativos (saber) que engloban el conocimiento del mundo, el conocimiento sociocultural y la consciencia intercultural, pero, también, tiene que adquirir destrezas y habilidades (saber hacer), la competencia existencial (saber ser) y la capacidad de aprender (saber aprender). Por todo ello, los planes de estudio han de ser interdisciplinarios e integrar la teoría y la práctica, fomentando el desarrollo de habilidades prácticas, la lógica, el análisis, la crítica. Saber utilizar la ciencia, la técnica y la teoría científica, para resolver problemas a través de una visión internacional y un pensamiento independiente.

También existen otro tipo de carencias que creemos que pueden repercutir en el proceso de enseñanza-aprendizaje de E/LE, tales como: a) no hay muchas posibilidades para hablar con gente nativa y practicar de forma eficaz el idioma; b) el tiempo que pasan los universitarios en los centros de multimedia es muy escaso; c) los estudiantes no suelen dedicar mucho tiempo a las actividades comunicativas fuera del aula; d) la ratio no es la más adecuada. En la mayoría de las clases hay muchos estudiantes, por ejemplo, en la clase de conversación y expresión escrita hay 20-25 estudiantes por grupo. Las clases que no son prácticas tienen aproximadamente 60-70 estudiantes, como por ejemplo las clases de gramática, lectura, cultura, compresión audiovisual, etc. Esto supone que la metodología de trabajo se apoye en el método tradicional (lección magistral) no favoreciendo el desarrollo de la competencia comunicativa intercultural, reclamada por las instituciones internacionales, lo cual nos habla de la brecha entre conocimiento académico y su uso práctico en el trabajo. La falta de interacción en el aula no favorece la competencia de aprender a aprender. En consecuencia, los Departamentos de Español deberían disminuir el número de estudiantes por clase para que los profesores puedan potenciar la competencia comunicativa intercultural, a través de actividades que supongan mayor interacción entre profesores-estudiantes y estudiantes-estudiantes.

En definitiva, la estructura curricular, el tiempo asignado y las metodologías son temas de debates urgentes. Los planes de estudio tienen que incluir una formación integral que tenga presentes los agentes sociales y comunicadores interculturales. También es necesario proponer una metodología ecléctica con el fin de establecer un contexto académico propicio para la excelencia en la enseñanza y en el aprendizaje. Asimismo, habría que motivar el interés del alumnado desde un diseño curricular más «práctico» y con materiales didácticos más interactivos. En este sentido, queremos destacar la propuesta de Sánchez Miguel y García-Rodicio (20I4) al plantear nuevas situaciones de lectura desde las nuevas tecnologías.

\section{Bibliografía}

Consejo de Europa (2002) Marco común europeo de referencia para las lenguas: aprendizaje, enseñanza, evaluación. Madrid: Ministerio de Educación, Cultura y Deporte.

Cortés, M. (20I2a) Nuestro currículo de español no es perfecto Universidad Fujen (Taiwán). SinOELE, 6, 84-IO2.

Cortés, M. (20I2b) La motivación por el estudio del español en Taiwán: primeros proyectos de investigación. SinoELE, 6, I-27. 
Huang, Y.-T. (2015) La enseñanza del español en Taiwán, la importancia del bloque cultura en los planes de estudio y el desarrollo de la competencia comunicativa intercultural (2009-2014). Tesis doctoral. Universidad de Valladolid. Valladolid.

Instituto Cervantes (2006) Plan curricular para la enseñanza del español como lengua extranjera. Biblioteca Nueva: Madrid. Recuperado el 20 de mayo de 20I4, de http://cvc.cervantes.es/ensenanza/biblioteca_ele/plan_curricular/default.htm.

Instituto Cervantes (2014) El español: Una lengua viva. Informe 20I4. España: Instituto Cervantes. Recuperado el is de agosto de 20I4, de http://eldiae.es/wp-content/uploads/20I4/ o $/$ El-espa\% $\mathrm{C}_{3} \%$ Biol-lengua-viva-20i4.pdf.

LIN, T.-J. (2OI2) La enseñanza del español en Taiwán: la necesidad del aprendizaje de segundas lenguas. Cuadernos Cervantes de la lengua española, 30, 9-15.

Mehrabian, A. (1972) Nonverbal communication. Chicago: Aldine-Atherton.

Moreno, F. y Otero, J. (2006) Demografía de la Lengua Española. Instituto Complutense de Estudios Internacionales (ICEI), 3, I-70.

Sánchez Griñán, A. J. (2006) Dificultades del enfoque comunicativo en China. En Actas del Primer Congreso Virtual E/LE. La enseñanza del español en el siglo XXI. Recuperado el 30 de febrero de 20I4, de http://congresoele.net/biblioteca/index.php?option=com_conte nt\&task $=$ view $\&$ id $=I I 5 \&$ Itemid $=48$.

Sánchez Miguel, E. y García-Rodicio, H. (20I4) Comprensión de textos, conceptos básicos y avances en la investigación actual. Aula. Revista de Pedagogía de la Universidad de Salamanca, 20, 83-103.

Vielma Vielma, E. y Salas, M. L. (2000) Aportes de las teorías de Vygotsky, Piaget, Bandura y Bruner. Paralelismo en sus posiciones en relación con el desarrollo. EDUCERE, año $3, \mathrm{n}^{\circ}{ }^{\circ}$, junio.

Vygotsky, L. (20Iо) Pensamiento y Lenguaje. Barcelona: Paidós.

Departamento de Estadística del Ministerio de Educación (Taiwán) http://www.edu.tw/Default.aspx?WID=3Id75a44-efff-4c44-a075-15ageb7aecdf.

Universidad Católica de Fujen http://www.span.fju.edu.tw/.

Universidad de Providence http://www.spanish.pu.edu.tw/main.php.

UNIVERSIDAD DE TAMKANG http://www.tfsx.tku.edu.tw/main.php.

Universidad de Idiomas Extranjeros Wenzao http://co24.wtuc.edu.tw/front/bin/home.phtml. 